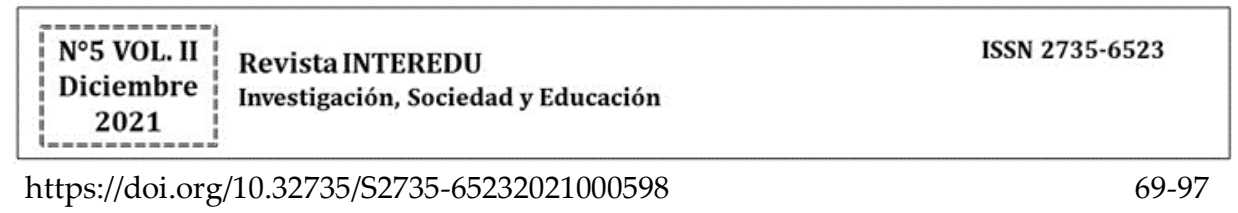

\title{
CONTEXTOS EDUCATIVOS EMERGENTES EN LA EDUCACIÓN INICIAL FORMAL
}

Emerging educational contexts in preschool education

\author{
DESIRÉE LÓPEZ DE MATURANA L. ${ }^{1}$ \\ Universidad de La Serena \\ gladys.lopezdematurana@userena.cl \\ https://orcid.org/0000-0001-6321-1073 \\ José Miguel VALENZUELA C. ${ }^{2}$ \\ Comunidades de Aprendizaje Territorio Coquimbo \\ jmvalenzuelacordova@hotmail.com \\ https://orcid.org/0000-0001-8683-9381 \\ BENJAMÍN ÁLVAREZ G. ${ }^{3}$ \\ Comunidad de Aprendizaje Territorio Coquimbo \\ benjamin.a01.g@gmail.com \\ https://orcid.org/0000-0001-5302-0927
}

\section{RESUMEN}

La investigación que da origen a este artículo ${ }^{4}$ es un estudio cualitativo en curso que pone el foco en el nivel de transición para caracterizar los contextos educativos emergentes en el ámbito escolar, toda vez que la

\footnotetext{
${ }^{1}$ Educadora de Párvulos, Dra. en Educación

2 Profesor de Historia, Magister (c) de Innovación y Didáctica

${ }^{3}$ Psicólogo, Licenciado en Sociología

${ }^{4}$ Proyecto Contextos educativos emergentes e inteligencia cultural en jardines infantiles en comunidad de aprendizaje de la comuna de Coquimbo. Financiado por la Dirección de Investigación y Desarrollo de La Universidad de La Serena en el marco del Proyecto PMI FIP ULS1505, Plan de Mejoramiento Institucional para generar cambios sustantivos en la calidad de la formación inicial de La Serena, que contribuyan al mejoramiento de la calidad del aprendizaje escolar en la región de Coquimbo.
} 
inteligencia cultural, social y la epistemología cotidiana de los niños y niñas quedan fuera del marco prescriptivo y encuentran su lugar en lo inacabado e indeterminado del currículum. La observación y registros de tipo etnográficos han permitido amplificar la escucha y la mirada de ese punto ciego donde encuentra asidero la cultura propia, los sentimientos, las emociones, la intuición y la percepción connotativa, con que niños y niñas dan sentido a la pedagogía. Su aprovechamiento puede ser una propuesta de acción que contribuya al cambio y a la mejora de la educación.

Palabras clave: Educación de la primera infancia; educación preescolar, epistemología; escuela; inteligencia y cultural; pedagogía.

\section{ABSTRACT}

This research has been driven by an ongoing qualitative study which has focused on preschool education in order to describe those emerging educational contexts in the school environment, whenever the cultural, social intelligence and daily epistemology of children set students outside the prescriptive framework for education, and so, they finally find their place in an unfinished and unclear curriculum for this level. Both the observation and the ethnographic records applied in this study have allowed us to amplify both, the listening and the look of this blind spot, providing a description of the place where the students' own culture coexist, and so, their feelings, emotions, intuition and connotative perception, as well as those other elements that together with children give true meaning to pedagogy. This research invites other researchers to consider this document as a source of relevant information, as it can contribute to the improvement of education.

Key words: Early childhood education; preschool education, epistemology; school; intelligence and culture; pedagogy. 
El reconocimiento de lo emergente en la educación ha dado paso a un cambio de paradigma que se prepara para dar un salto desde la búsqueda de certezas a la aceptación de la incertidumbre y, por consecuencia, el papel docente ya no representa la razón absoluta, lo invita a redireccionar el trabajo en aula para incorporar lo imprevisto, transformando los tiempos y espacios educativos en escenarios para intercambiar significados, hacer representaciones simbólicas del mundo, produciendo sentidos e intersubjetividad.

El interés por investigar el vacío que ocurre entre los conocimientos escolares y los cotidianos de los niños y las niñas que asisten a la educación inicial, surge de una investigación previa, desarrollada en la Universidad de La Serena, con fondos de la Dirección de Investigación y Desarrollo (DIDULS, 2016), cuyo propósito fue caracterizar la propensión a aprender y el desarrollo de las funciones cognitivas de los niños y las niñas que asistían a niveles iniciales de educación. Los registros etnográficos dieron cuenta que cuando los niños y niñas creaban sus actividades, por lo general lúdicas, se ponían en exposición directa a estímulos que les exigían, como respuesta, funciones mentales superiores, entre otras, la abstracción, representación, anticipación y combinación, todas ellas manifestaciones operativas de sus funciones cognitivas y de sus experiencias previas.

La Educadora está reforzando los contenidos sobre las partes del cuerpo humano 5

Docente: apuntando sus piernas, dice: “¿y esto cómo se llama?"

Yeison: "iLas patas!”

Docente: “'Las piernas, bien!"

Omar: "iLos animales tienen patas, las personas tenemos pies!"

Docente: "iLos pies, cierto!"

Omar: " $¡ Y$ Yo he visto a una persona que le falta una pierna!"

Docente: “AAhora lo que nosotros estamos haciendo ahora...!"

${ }^{5}$ Los registros describen tal y como se producen las situaciones y los diálogos son textuales.

INTEREDU № 5 VOL. II (DicIEMBRe 2021) PÁGS. 69-97. ISSN: 2735-6523| 71 
Yeison: "Es un abuelito!"

Docente: “¡A ver!"

Omar respondiendo a Yeison: “SSí!”

Yeison: "iAllá afuera hay un abuelito que vende corta uñas que no tiene piernas!"

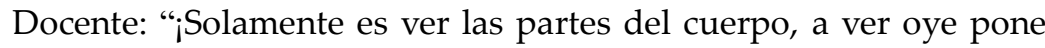
atención!" le dice a Yeison.

Yeison se gira y mira a la profesora.

Los niños y las niñas atendían a más de una fuente de información: a) al contenido trabajado por la educadora durante esa unidad temática, b) a la pregunta que ella realizaba para reafirmar lo aprendido y, c) a la relación análoga que se representaban mentalmente. La pregunta surgió del propósito educativo que la educadora había prescrito, y lo que resultó como respuesta, fue la relación que Omar estableció con una situación propia de su vida cotidiana. Sin embargo, en el contexto de lo prescrito, podríamos decir que se consideró fuera de marco, pues, aunque esa respuesta abría el diálogo y situaba la actividad, haciéndola más pertinente $y$, permitía expandir la situación educativa y de aprendizaje, no se consideró apropiada, por lo mismo, fue desechada con un llamado a la concentración. Ese momento, develaba el contexto educativo cotidiano y cultural, que niños y niñas compartían y que reconocieron como lugar común, en contraposición al contexto escolar, donde la educadora continuaba imponiendo lo prescrito, negando con ello el acervo cultural familiar y comunitario. La recurrencia de este tipo de situaciones fue un hallazgo que, si bien no era el propósito de dicha investigación, provocó el interés por indagar esos contextos educativos paralelos que daban cuenta de la inteligencia cultural de los párvulos.

Para llevar a cabo este estudio se estableció una alianza con Comunidades de Aprendizaje - Territorio Coquimbo, en cuyo seno, participan las escuelas donde se realizó el trabajo de terreno y los grupos focales con las y los agentes educativos de los mismos establecimientos. Dicha entidad sustenta su acción educativa en el aprendizaje dialógico, 72 | INTEREDU № 5 VOL. II (DiCIEMBRE 2021) PÁGS. 69-97. ISSN: 2735-6523 
donde la inteligencia cultural se entiende en interacción con otros principios como el diálogo igualitario, la transformación, la dimensión instrumental del aprendizaje, la creación de sentido, la solidaridad y la igualdad de las diferencias para la mejora de los aprendizajes de las comunidades educativas. A saber, el diálogo igualitario se entiende como una acción humana que parte en una conversación entre personas y que está orientado al consenso que se produce en relación a la validez de los argumentos que se despliegan en esas conversaciones cotidianas; la inteligencia cultural, aunque es un concepto complejo de definir por su esencia multifactorial y cultural, se entiende como la inteligencia del mundo de la vida, de la cultura y la comunidad. Es decir que todas las personas somos capaces de aprender y transferir conocimientos mediante la interacción con otros y otras, por el hecho de pertenecer a una cultura; el principio de transformación, favorece en las personas la generación de herramientas personales, emocionales, cognitivas y técnicas para mejorar sus condiciones de vida e incidir en las transformaciones sociales y en sus condiciones de existencia; la dimensión instrumental articula ciencia y utopía. En este sentido busca que todas las personas tengan acceso a los conocimientos y competencias más relevantes de la sociedad actual (aritmética, lenguas, conocimientos comunicacionales, tecnológicos, saberes de ecología y cuidados del ambiente, democracia etc.) que permitan mejorar las condiciones de vida de los grupos sociales. En este sentido, se hace relevante que las personas puedan tener acceso a una mejor educación, para que puedan disputar mejores puestos de trabajo y mejorar las condiciones democráticas y ecológicas de vida; la creación de sentido, apunta a posibilitar un tipo de aprendizaje que parta de la interacción y de las demandas propias de las personas, y desde esos sueños trabajar conjuntamente para hacerlos realidad; el principio de solidaridad, se entiende como la práctica humana promovida como práctica política para asumir un compromiso más igualitario y democrático en los centros educativos, transferible a otros ámbitos de la sociedad. Rompe con la lógica del asistencialismo ante la carencia, proponiendo espacios democráticos pensados para el bien común $\mathrm{y}$, finalmente, el principio de Igualdad de la diferencia, plantea que todos INTEREDU № 5 Vol. II (DiCIEMBRE 2021) PÁGs. 69-97. ISSN: 2735-6523| 73 
Desirée López de Maturana, Miguel Valenzuela y Benjamín Álvarez

tenemos el mismo derecho de conocer, en un marco de respeto y dignidad para fortalecer y acelerar los aprendizajes. La diversidad genera un marco de interacciones nutricias para la calidad educativa y promueve el derecho de vivir de forma diferente y ser respetados(as) en nuestra digna decisión religiosa, política, sexual, etc. (Aubert, et al., 2010)

\section{SÍNTESIS TEÓRICA}

\section{CONTEXTOS EDUCATIVOS EMERGENTES}

El conocimiento escolar, por lo general, surge de la información que arrojan la experimentación y la observación en condiciones creadas científicamente para ello, por lo que está dotado de sistemas explicativos técnicos y principios deductivos para la resolución de problemas, a diferencia de los conocimientos cotidianos que se construyen en escenarios que demandan más de la comprensión de situaciones para la elaboración de procedimientos, formas de actuación y explicaciones propias que responden a un modelo mental implícito. Es decir, la consideración de estas teorías subjetivas, permiten hacer referencias a las hipótesis que elaboran los sujetos, para dar sentido a su entorno y para actuar en él. Si bien éstas pueden no responder a una ciencia, tienen la riqueza que radica en su carácter metafórico, que surge en virtud de una analogía entre el saber "lego" y el saber de la ciencia (Catalán, 2010; Catalán y Cuadra, 2016). Esta relación entre saberes cotidianos y científicos crea otros contextos educativos que son los que hemos llamado, emergentes. Otorgar un sentido y relevancia al conocimiento cotidiano en relación al conocimiento escolar, es reconocerlo como un ingrediente fundamental en la construcción de este último. De este modo, es dable pensar que, si los seres humanos estamos elaborando permanentemente teoría cotidiana, aun careciendo de herramientas y condiciones necesarias para elaborar teorías científicas, es posible que las teorías cotidianas estén en el origen o a la base de las teorías científicas. Visto desde una perspectiva ética, pudiera pensarse que son precursoras 74 | INTEREDU № 5 VOL. II (DiCIEMBRE 2021) PÁGS. 69-97. ISSN: 2735-6523 
a las teorías científicas a las que adscriben y a las elaboraciones científicas que puedan surgir (Catalán, 2010).

Varela (2000) plantea como uno de los puntos clave de la emergencia, que la mente existe y no existe y, que por esta cualidad difícil de explicar ha sido por lo general, incomprendida o reducida en su definición. También señala que cuando más observamos el funcionamiento del proceso de cognición, se evidencia la participación de componentes muy individuales, neuronas o grupos de neuronas, o poblaciones de neuronas y que es a partir de estas reglas locales que se produce la emergencia de un nivel global. Esta "tiene un status ontológico diferente, porque trae consigo la creación de un individuo, o de una unidad cognitiva".

La mente no es la representación de un determinado estado de cosas; la mente es la producción constante de esta realidad coherente que constituye un mundo, un modo coherente de organizar las transiciones locales-globales. Dicho de otra manera, así como la percepción es imaginaria, la imaginación se basa en la percepción [... Esto representa un gigantesco paso conceptual, desde la idea que existen propiedades del mundo que hay que aprender para formarse una imagen adecuada de la realidad, hasta la noción de que prácticamente cualquier cosa nos sirve de excusa para, inventar la realidad. Nuestro mundo es imaginación y fantasía, y es por esto que es de fundamental importancia que los niños desarrollen el suyo (p.3-8).

Gopnik (2010) hace referencia a la maravillosa capacidad de pensamiento contrafactual que se manifiesta mediante la imaginación, la esperanza y la hipótesis, que permiten alterar un aspecto de un hecho pasado o de un posible futuro y anticipar las consecuencias de ese cambio. Con ello nos señala que no sólo vivimos en este mundo, sino que vivimos en un universo de muchos mundos posibles, que podemos llamar sueños, ficciones o hipótesis. Ante las dudas que surgen de los científicos convencionales, que sustentan sus teorías en los planteamientos de Freud y Piaget, quienes señalan que los bebés y los niños pequeños se limitan al 
aquí y al ahora, a sus sensaciones, percepciones y experiencias inmediatas y que aun cuando están imaginando y fingiendo no distinguen entre realidad y fantasía, porque esta sería otra clase de experiencia inmediata, ella refuta diciendo:

Hemos averiguado que incluso los niños muy pequeños son capaces de considerar posibilidades, de distinguirlas de la realidad e incluso de utilizarlas para cambiar el mundo. Son capaces de imaginar otras diferentes formas en que podría ser el mundo en el futuro y utilizarlas para hacer planes. Son capaces de imaginar formas diferentes que el mundo podría haber tenido en el pasado y reflexionar sobre posibilidades pasadas. Y lo que resulta aún más espectacular, pueden crear mundos totalmente imaginarios, disparatadas ficciones y fingimientos asombrosos [...] Esos increíbles mundos imaginarios son una parte conocida de la infancia [...] sin embargo las nuevas investigaciones están cambiando profundamente, la idea que teníamos de esos mundos (p.29).

Una situación que frecuentemente se percibe en el ámbito escolar se caracteriza por el desencuentro de expectativas entre la información entregada, de manera unidireccional a los niños y niñas $\mathrm{y}$, sus reales problemas, requerimientos, necesidades, formas de aprender, descubrir y generar conocimiento propio. Se castiga o se premia según se ajusten al comportamiento esperado, pero esta capacidad para crear nuevos mundos, les permite captar el mensaje y comprenden que deben fingir, aprendiendo desde la más temprana infancia, que deben demostrar que saben lo que no saben y que son lo que no son, generando una discrepancia entre la imagen interior y la construida para satisfacer las expectativas de un sistema exógeno. Lo importante es que esa identidad propia existe, está en permanente construcción, y con cierta frecuencia late y resuena internamente para manifestarse, a veces de maneras consideradas disruptivas (Wolf, 1994). 


\section{PEDAGOGÍAS INVISIBLES}

En ese mismo sentido, Acaso (2018) plantea la importancia de poner atención a las pedagogías invisibles, que de acuerdo a su definición serían la parte connotativa del discurso educativo explícito, como elaboración cultural subjetiva del mismo. Hacer hincapié en este aspecto de las interacciones, permite a los y las docentes mirar su praxis con este prisma y desarrollar una hermenéutica de la sospecha, mediante acciones semióticas para, no sólo analizar y reflexionar sobre su práctica, sino para cambiar lo que no funciona. En este sentido es importante comprender la práctica docente como un conjunto infinito de microdiscursos, que los niños y las niñas interpretan adaptándose a los mensajes o transgrediéndolos, según sean sus herramientas psicoemocionales y cognitivas. La autora sostiene que:

El pensamiento se maneja con representaciones y que estas están siempre conectadas con otros signos, en una cadena infinita de relaciones o semiosis y que incluso la percepción será comprendida dentro de ese mundo representacional. Cuando el destinatario tiene que interpretar, se valdrá de lo que ve que ha sido expuesto en forma de enunciado y tratará de reconstruir la intención y el sentido que la situación porta. (p.47)

Es decir, que cada acción no sólo dice algo, sino que transforma a quien la realiza, dice y hace; deja entrever lo que somos o lo que deseamos ser. En general, nos falta percibir que nuestras conversaciones van más allá de lo expresado con palabras y que pueden ser una herramienta fundamental para esos cambios de estados de ánimo de otras personas, y que al hacerlo podemos abrir o cerrar nuevos canales de acción (Echeverría, 2005). A partir de la categorización de los distintos sistemas de representación humanos (visual, auditivo, kinestésico, emotivo, racional) podemos ver como desde cada uno de ellos emergen discursos que afectan a las dinámicas del acto pedagógico de manera profunda $y$, debemos reflexionar sobre ese proceso de recorrido del discurso, que si bien tiene como destinatarios(as) a los y las estudiantes, el proceso no se 
Desirée López de Maturana, Miguel Valenzuela y Benjamín Álvarez

acaba ahí, porque es un continuo, dinámico y complejo. En este sentido y siguiendo con la idea de la autora, es un imperativo para la docencia hacerse cargo de las pedagogías invisibles, teniendo en cuenta que la docencia que se sustenta fundamentalmente en el rol técnico, acata el punto de vista de una realidad que deciden otros, mientras que aquella que da espacio a la creación, tiene un punto de vista propio y el proceso de transformación de la realidad, será reflexionado, comprometido y ético.

\section{LENGUAJE Y ACCIÓN COMUNICATIVA}

El protagonismo del lenguaje en nuestra vida humana es innegable. En palabras de Maturana (2001), los seres humanos nos constituimos en el lenguaje. Sin embargo, esta prioridad que le asignamos no opaca la existencia de otros dominios relevantes en la compleja acción comunicativa, toda vez que, para comprenderla, debemos poner atención al dominio del cuerpo y de la emocionalidad. Cada dominio tiene sus propios fenómenos constitutivos, que no pueden reducirse a un dominio diferente al suyo, pero sí reconocerse en las relaciones de coherencia, donde lo que acontece en uno de ellos condiciona lo que sucederá en el otro. Posturas físicas, emocionalidad y lenguaje se comportan y se influencian entre sí de manera congruente. Sin embargo, no es tan obvio que estos tres dominios mantengan esta coherencia y sería necesario mostrar por qué sucede aquello y cuáles son los mecanismos con los que se produce la influencia de un dominio sobre el otro. Al existir coherencia entre los dominios, nos permite utilizar cualquiera de los tres, para referirnos a los otros dos. Esto nos permite decir que podríamos realizar una "reconstrucción corporal" de un determinado juego de lenguaje o acometer una "reconstrucción lingüística" de una determinada emoción.

Gabriela Mistral en la carta a su madre ausente aborda, de manera poética y autobiográfica esa sensibilidad que, a muy temprana edad, aun sentada en las piernas de su madre, podía leer y reconocer en su rostro las 
emociones que le embargaban. Podía ver y dar significado a cada arruguita y aprender de cada gesto. Ella dice:

...Yo jugaba con tus cabellos como con hilillos de agua escurridizos, con tu barbilla redonda, con tus dedos, que trenzaba y destrenzaba. Tu rostro inclinado era para tu hija todo el espectáculo del mundo. Con curiosidad miraba tu parpadear rápido y el juego de la luz que se hacía dentro de tus ojos verdes; iy aquello tan extraño que solía pasar sobre tu cara cuando eras desgraciada, madre!

Sí, todito mi mundo era tu semblante; tus mejillas, como la loma color de miel, y los surcos que la pena cavaba hacia los extremos de la boca, dos pequeños vallecitos tiernos (Scarpa, 1978, párr. 8)

Feuerstein plantea que la esencia de la Inteligencia no radica en el producto mensurable, sino en la construcción activa del individuo que tiene capacidad para usar experiencias adquiridas previamente y ajustarse a nuevas situaciones o transformarlas (López de Maturana, 2010). Podríamos decir que nuestros ambientes o nuestras asociaciones pueden influir en nuestras elecciones, pero eso no quiere decir que las determinen. Al respecto, Madrona (2014) sustentado en los planteamientos de Danah Zohar, dice que el ser humano más que estructurar una información en relación a si mismo o de su entorno, la interpreta, porque la fija como ideología, como carácter, actitud, objetivos o esperanza en un yo. Es decir que, la naturaleza humana es funcional y circunstancial en el proceso de crecimiento y define una realidad entre todas las posibles maneras de concebirla, de acuerdo a las circunstancias.

Según Goldberg (2006), la competencia es una especial habilidad para reconocer las similitudes entre problemas aparentemente nuevos y problemas ya resueltos. Esto implica que la persona competente debe tener a su disposición un gran conjunto de representaciones mentales o, podríamos decir, inteligencias, para que con cada una de ellas capte la esencia de un amplio abanico de situaciones específicas y de las acciones más eficaces asociadas con esas situaciones para dar su mejor respuesta. Para ello debemos hacer visible y lidiar con lo que Elisworth (Acaso, 2018) 
Desirée López de Maturana, Miguel Valenzuela y Benjamín Álvarez

denomina el tridiálogo, para referirse a todo aquello que existe entre el o la Educadora y los niños y niñas, como son: los recuerdos, los deseos, los tabúes, los miedos y el placer, que la pedagogía tradicional nos ha enseñado que no deben intervenir, pero que como retorno reprimido termina perturbando y complejizando aún más, la acción pedagógica.

\section{ROL DOCENTE E INTELIGENCIA CULTURAL}

La necesidad epistémica, a la que hemos aludido, se despliega plenamente en la propensión a aprender del ser humano y se convierte en un imperativo para que los y las docentes pongan mayor atención en qué y cómo se lleva a cabo la tarea educativa, considerando en ese análisis las dimensiones afectiva, social y cultural, para descubrir nuevas formas de pensar y re-contextualizar constantemente los propios modos cognitivos. Generar nuevos contextos de y para la reflexión es ineludible, porque la transformación epistemológica va a depender del contexto desde el cual dicha reflexión emerge (Bateson, 1979). Ampliar la mirada para situar la reflexión abre paso al diálogo igualitario como práctica comunicativa, que tiene en cuenta todas las aportaciones que hacen las personas que participan de esa instancia. (Aubert, et al., 2010), consideran estos elementos, esenciales de la organización humana basada en el diálogo y el consenso, como una ruta plausible para superar las desigualdades y avanzar hacia la transformación social.

[...] cuando planificamos la enseñanza en función de las diferencias individuales o grupales del alumnado, viendo sólo que son de etnia gitana, inmigrantes o de familias pobres, no académicas, etc. Entonces reducimos sus objetivos educativos, con lo que acaban aprendiendo menos que aquellos y aquellas cuyas diferencias no les ponen en riesgo de exclusión social (p.223).

Al asumir la tarea docente estamos aceptando que somos nosotros(as) los(as) responsables de nuestros currículums y, por lo tanto, de decidir el qué, el para qué y cómo hacer para que los contenidos 80 | INTEREDU № 5 VOL. II (DiCIEMBRE 2021) PÁGs. 69-97. ISSN: 2735-6523 
cumplan el propósito educativo, con creatividad y coherencia contextual. López de Maturana (2010) destaca la importancia de establecer relaciones significativas con los niños y niñas que pueden ser catalogados(as) como privados culturales en un contexto educativo formal, que sólo utiliza estrategias educacionales estimulantes y pertinentes al medio en el que ellos y ellas viven, pero no les ayudan a traducir esos recursos para que le sean de utilidad en su propio proceso de aprendizaje.

Esto cobra vital importancia ante el acecho permanente de la "pedagogía tóxica" (Acaso, 2018), cuya postura técnica, funcional y operativa que acata y aplica lo establecido, nos atrapa y nos aleja progresivamente de la posibilidad de crear. Lo más grave, es que nos aleja del contexto epistemológico y de la inteligencia cultural de los niños y de las niñas. Pero no es lo único a lo que debemos poner atención, según la autora, si no nos hacemos cargo de las pedagogías invisibles que desarrollamos, todo lo que vayamos a hacer, será crear para los demás "[..] acabaremos trabajando a partir de libros y textos de otros, de unidades didácticas de los otros [...] acabaremos trabajando con y para las ideas de otros" (Acaso, 2018, p.61). En esta misma línea reflexiva, Calvo (2014) hace una relación entre aprendizaje y establecmiento de relaciones posibles, distinguiendo entre los tiempos educativos formales cronológicos y los cotidianos o informales históricos, afirmando que:

El educador inmerso en el tiempo histórico puede innovar en la medida que comprende que los hechos pueden tener muchas explicaciones y no una sola. En cambio, el profesor no educador que transita por el tiempo cronológico solo será repetidor, pues nunca comprenderá que la educación no es repetición de relaciones prestablecidas, sino creación de relaciones posibles (p.58).

La acción de dar clases, la podemos asumir desde estas dos perspectivas, la que reproduce y transfiere información y la otra, que a partir de esta información crea una narrativa, vivencial, metafórica y transformadora. La segunda opción, permitirá el desarrollo de habilidades y estrategias didácticas más apropiadas para abordar las demandas de una sociedad qué, de manera progresiva, está transitando a INTEREDU № 5 Vol. II (DiCIEMBRE 2021) PÁGs. 69-97. ISSN: 2735-6523| 81 
Desirée López de Maturana, Miguel Valenzuela y Benjamín Álvarez

una organización basada en el diálogo como forma de tomar decisiones, despojándose de prácticas posicionadas en el poder, para favorecer interacciones y relaciones interpersonales más democráticas, cuyos importantes pasos irán sustituyendo el argumento de la fuerza por la fuerza de los argumentos (Aubert et.al., 2010).

$\mathrm{Si}$ pretendemos superar la desigualdad que genera el reconocimiento exclusivo de las habilidades académicas y la exclusión de aquellas personas que no tienen acceso al procesamiento de la información, debemos reflexionar sobre qué tipo de habilidades se están potenciando en los contextos formativos y, si con ello, se facilita la interpretación de la realidad desde una perspectiva transformadora (p.187).

La educación centrada en el diálogo permite llegar a acuerdos y democratizar las opiniones para dar espacio a las posiciones propias e individuales, en oposición al poder estratégico tan común en la educación formal, de llevar a los niños y niñas a responder lo "adecuado" para el campo epistemológico de la escuela o de la o el educador(a). Freire (1997) interpela a los y las docentes preguntando, ¿cómo puedo ser tolerante si, en vez de considerar al otro como diferente de mí, lo considero como inferior...? Esta pregunta/ reflexión es fundamental para entender el principio de igualdad de las diferencias. Situación que no es posible, cuando creemos que existen culturas que son inferiores a otras. En ese contexto dejamos de creer y bajamos las expectativas, ponemos menor complejidad en los procesos de aprendizaje, porque el poder de la creencia de que el otro $u$ otra es inferior, nos hace actuar de acuerdo a ello y podemos llegar a convencerles que no son capaces de realizar determinadas acciones, por lo general cognitivas, debido a su procedencia o su cultura.

Generar estos ambientes educativos respetuosos es considerar como principio esencial la inteligencia cultural, ya que esta aloja en su centro la idea de que todas las personas tienen la capacidad de ejercer una 
acción y de construir sus propias reflexiones, que van surgir de las relaciones que establezcan con sus propios contextos de proveniencia. Asume también, que no solo existe la inteligencia académica y que también existen otras formas de inteligencias, pero que su gran disputa es con las teorías del déficit, orientadas a la generación de etiquetas que perpetúan las desigualdades y fortalecen la visión hegemónica de la inteligencia académica como más importante que otras. La inteligencia académica es importante, pero así también lo es la inteligencia social, comunicativa, tecnológica, etc. [...] no es cuestión de tener o no tener inteligencia académica, de ser o no ser inteligente académicamente, sino que se trata de algo que se aprende y que siempre es modificable y mejorable mediante la participación en actividades educativas. [...] la inteligencia es un potencial cognitivo (Aubert et al., 2010).

\section{APRENDIZAJE DIALÓGICO Y ESTABLECIMIENTO DE RELACIONES}

Lo anterior, se trata de un problema de acceso y de las formas de cómo llegamos a generar conocimiento y esto es un proceso que se puede trabajar desde un enfoque dialógico. López de Maturana (2010) señala que las escuelas deben transformarse en ambientes activo-modificantes en contraposición a los ambientes pasivo-aceptantes, que por lo general son promovidos por una cultura escolar que concibe el proceso educativo como unidireccional, vertical, pasivo y carente de significado. En tanto los ambientes activo-modificantes, generan relaciones multidireccionales entre los(as) docentes, niños, niñas y los estímulos.

López de Maturana (2014) señala que "los elementos pedagógicos utilizados por los profesores en las clases y la forma en que realizan la docencia impactan sustantivamente en las relaciones que establecen con sus estudiantes y cómo estos se involucran en la tarea de aprender" (p.281). La misma autora señala que entre los(as) docentes que han sido reconocidos por sus pares como buenos(as) profesores(as) existen algunos patrones comunes, como reconocer que incentivan a sus estudiantes usando estrategias para hacer más amena y divertida la clase. Uno de ellos dice, "les toco muchas cosas de su vida real, les facilito el camino, les hago 
ameno el aprendizaje; en mis clases soy una persona optimista y positiva, demuestro que me gusta lo que hago" (p.289). Otro aspecto importante que señala este docente, es que en las clases refuerza la seguridad de niños y niñas y su sentimiento de ser competentes, orientando su trabajo, no sólo al aprendizaje de los contenidos de las distintas asignaturas, sino a que sean capaces de integrarlas en un macro ámbito social, al que pertenecen y conocen mejor.

Taleb (2011) en El cisne negro desarrolla ideas que se relacionan directamente con las contradicciones encontradas habitualmente en los tiempos-espacios educativos formalizados. No es extraño ver que un suceso se produce precisamente porque no se esperaba que fuera a ocurrir y que, por lo mismo, lo que creemos saber, estratégicamente, puede ser completamente intrascendente, porque la incapacidad de predecir las rarezas implica la incapacidad de predecir el curso de la historia, en ello radica la importancia de reconocer aquellos elementos que subyacen a los hechos y que permiten su autoorganización. Muchas veces, tal como ya hemos señalado, estas relaciones culturales que los niños y niñas establecen no se ven, porque son situaciones emergentes cuyo contenido está dado por experiencias que ocurren en su vida fuera de la escuela y cada vez que se da la oportunidad surge con total naturalidad, rompiendo el statu quo del ambiente escolar. Son el constructo experiencial significativo y relevante que provienen de las culturas populares (Bruner, 1999) que dan vivacidad a la experiencia educativa y que no tiene que ver con adaptar el nuevo conocimiento al contexto previo, sino que, a partir de esos mundos de la vida, dar sentido a lo nuevo. Según plantea Varela (2000), uno de los más importantes avances en ciencia en los últimos años es la convicción de que no podemos tener nada que se asemeje a una mente o a una capacidad mental, sin que esté totalmente encarnada o inscrita corporalmente, envuelta en el mundo y que surge como una evidencia inmediata, inextricablemente ligada a un cuerpo que es activo, que se mueve y que interactúa con el mundo. 
La situación radica en la estructura de nuestra mente, que no aprende reglas sino hechos y el "suceso raro" equivale a la incertidumbre. No abordar o no considerar esta brecha epistemológica, puede ser un impedimento para generar espacios educativos afectivos, constructivos, y ricos en aprendizaje. Es imposible dialogar si profesores y estudiantes hablan en frecuencias distintas, por ello se deben remirar el interior del aula como un espacio y tiempo relacional, que puede llegar a ser cualitativamente dialógico, siempre y cuando interactúen las distintas dimensiones que afectan la construcción de significados en relación con los contenidos o la realidad escolar. El aprendizaje dialógico apela a la igualdad en la diferencia, donde lo diferente es, precisamente, lo que enriquece las experiencias de aprendizaje. La escuela tradicional, por lo general, no da lugar a estos tiempo-espacios para el reconocimiento del otro(a) como personas activas, individuales y sociales, desconociendo que la construcción de saber, posee esta doble dimensión. En este tránsito permanente entre lo formal y lo cotidiano, o entre una realidad y otra, los niños y niñas van respondiendo a situaciones del contexto con fluidez cognitiva y socio afectiva, evidenciando la propiedad multifactorial del aprendizaje (DIDULS, 2016). Con estas ideas podemos reforzar que, el diálogo igualitario, permite a las personas fundamentar desde la fuerza de sus propios argumentos, que son los que van a dotar de validez su hablar y su decir. En este caso los argumentos que usan los niños y niñas en relación a su juicio ético, a veces resulta más coherente que el actuar del mundo adulto que los rodea.

A lo anterior, es dable agregar también, lo que señala L'Ecuyer (2012), cuando hace hincapié en las formas que niños y niñas van mostrando que aprenden por medio de su interés permanente por explorar, por poner a prueba, por hipotetizar y comprobar, porque disfrutan del asombro, del desafío, de lo diferente, y por lo general, de todo aquello que transgrede lo establecido. 
Desirée López de Maturana, Miguel Valenzuela y Benjamín Álvarez

\section{METODOLOGÍA}

La investigación es de índole cualitativa, descriptiva e interpretativa e incorpora grupos focales y herramientas etnográficas, como la observación y los registros de campo para la recogida de datos. Se utilizó un modelo de análisis descriptivo sustentado en la teoría fundamentada (Krause, 1995), se realizó su estudio producto del levantamiento de categorías emergentes o códigos semánticos, los que permitieron resumir, en un concepto, los elementos esenciales de las situaciones registradas como las interacciones, los diálogos de niños, niñas, docentes y otras manifestaciones que fueron surgiendo, en tiempos y espacios educativos dentro de la escuela, para generar la información necesaria.

El plan de análisis de registros, tiene como finalidad la generación de teoría, que se va produciendo desde el momento mismo de la recogida de información en el campo y que se va nutriendo y consolidando por medio del análisis y las definiciones conceptuales de las categorías. La metodología requirió realizar algunos pasos indispensables como la codificación semántica o abierta, la codificación axial y la codificación selectiva de los datos, para dar sustento a dicha teoría y a las conclusiones finales.

Esto ha permitido ir caracterizando los contextos educativos emergentes que los niños y niñas generan haciendo uso de su inteligencia cultural para traducir los contenidos y experiencias escolares. Para ello, hemos utilizado la metodología de análisis del discurso, específicamente en aquellos aspectos auxiliares del lenguaje que cumplen una función expresiva y eminentemente comunicativa, lo que nos ha ayudado a interpretar y comprender cómo pueden estar pensando los niños y niñas, qué funciones del sistema lingüístico utilizan para representarse la realidad, qué estamos considerando y traduciendo de los modos de comunicación utilizados por ellos(as) en las interacciones escolares y cómo son sus reacciones gatilladas por la lectura fina que hacen del contexto general y del educativo. Del mismo modo, tuvimos que considerar la indeterminación para dar un espacio y tiempo relevante al asombro y al descubrimiento, todos ellos, elementos esenciales de la 86 | INTEREDU № 5 VOL. II (DiCIEMBRE 2021) PÁGs. 69-97. ISSN: 2735-6523 
emergencia. En palabras de Peirce (Acaso, 2018), la idea es sintonizar con lo que ven y traducen las y los niños de ese entorno. De allí surgen algunas categorías relacionadas teóricamente con los microdiscursos y las pedagogías invisibles. Veamos algunas situaciones registradas, breves pero ricas en mensajes, representaciones y simbolismos:

- IPA19EP1 11/09/2019

Dos compañeros se miran entre si y uno pregunta, ¿eres mi amigo? el otro asiente y se abrazan.

- IPA19AP2 25/09/2019

Dos compañeros pelean.

Docente: "¡No a los golpes Adolfo"!

Niño 1: "¡suéltame! ¡Yo no soy su empleado!"

Niño 2: “'Sí!, ¡si eres su empleado!”

\section{CATEGORÍA: MicRODISCURSOS}

En ambos casos, la categoría o codificación selectiva es el microdiscurso. A la luz de su definición hemos podido interpretar que el abrazo surge, probablemente, de un gesto y una mirada que quien la recibe le atribuye la connotación de amistad, pero lo lleva a preguntar y comprobar si esa interpretación es o no correcta y luego al corroborar la reciprocidad, sella el momento con un abrazo. Cuando esto sucede, se devela otro mensaje, donde el abrazo marca la diferencia entre los que son amigos y los que no lo son.

Podemos ver en esta acción que reconocer para sí, un gesto de amistad en otro, genera una modificación conductual - emocional y el abrazo consolida la coherencia de ese diálogo que se inicia sin palabras, por medio de un campo de intervenciones indirectas. Estos campos son complejos e indeterminados y propicios para los contextos educativos emergentes. La nítida capacidad de los niños y niñas de leer esos mensajes, les vuelve mejores receptores de estos fenómenos que van creando distintos estados de ánimo, sin razón aparente. 
Desirée López de Maturana, Miguel Valenzuela y Benjamín Álvarez

\section{CATEGORÍA: APRENDIZAJE EXPERIENCIAL - MICRODISCURSO}

Así también, el otro registro muestra el trato violento y asimétrico en la relación asociada, a ser o no ser empleado de otro. Esta experiencia, además, tiene relación con otra categoría "aprendizaje experiencial" que, desde el aprendizaje dialógico, se define como el testimonio de la presencia del mundo social y de la inteligencia cultural de cada persona y de cada comunidad. Se relaciona con el mundo de la vida, que dialoga permanentemente con el presente y forma parte de las interacciones que cada niño y niña desarrolla. Flecha (2009) plantea al respecto, que "Nuestro yo no está nunca solo, tampoco el de nuestros alumnos y alumnas. Siempre están las amistades, las pareja, profesores y profesoras, familiares, etc. dentro nuestro" (p. 2). En este sentido podemos decir que cada quien es un agente social que da cuenta de la forma en que mira, vive y transmite su mundo. Cuando el niño habla sobre el ser o no ser empleado de otro, habla desde ese saber construido cultural y socialmente, desde los principios y valores que habita. El rol del empleado es algo que le pertenece a una comunidad y que representa esa inteligencia cultural vivida y heredada por generaciones.

Siguiendo con la misma categoría, en los siguientes registros vemos como junto a los microdiscursos, emerge la "analogía" como herramienta de comunicación y traducción de la realidad:

- IPA19AP4 03/10/19

(Adolfo está dentro de la sala, no saluda, está castigado, tiene que terminar su trabajo)

Etnógrafo: ¿qué te pasó?

Adolfo: nadie quiere ser mi amigo, porque me porto mal. ¡Quiero salir al patio...!

¡mi hermano dice garabatos portándose bien!, [repite] ¡mi hermano dice disparates cuando se porta bien! 


\section{CATEGORÍA: RELACIÓN ANALÓGICA}

Adolfo no comprende por qué un comportamiento que ocurre habitualmente en su entorno cotidiano y que no genera conflicto, cuando él lo reproduce en la escuela es castigado. Él dice: "¡mi hermano dice garabatos portándose bien!”, es decir que asocia el castigo al mal comportamiento, entonces cuando su hermano dice garabatos y no es castigado, entonces es parte de un comportamiento normal. Estos dobles mensajes, confunden a los niños y niñas, toda vez que esta forma de hablar es propia de su entorno cotidiano y, en este caso, no sólo él está recibiendo el castigo en la escuela, porque de acuerdo a esta lógica, también lo recibe su familia, sus amigos, amigas y su comunidad. Si esto forma parte de su inteligencia cultural y no encuentra un espacio de diálogo, de reflexión colectiva, de respeto, sino por el contrario, sólo el castigo, debemos pensar, ¿cuál será entonces, la explicación que hará sobre este hecho?

\section{- IPA19AP4 03/10/19}

En el mismo momento del registro anterior, otro niño le habla al etnógrafo:

Pedro: ¡su bufanda tío! (refiriéndose al etnógrafo), mi mamá le tiene muchos vestidos a mi hermana, para que se vea linda. ¿Tiene frio? ¡Yo no quiero que se resfríe!

En este caso, el niño establece una relación estética entre la bufanda y los vestidos de su hermana (analogía). Dice que su mamá le tiene muchos vestidos para que se vea linda, podemos interpretar que, además de reconocer la utilidad práctica de la bufanda, la aprecia estéticamente y esa apreciación la comunica indirectamente por medio del paralelo o analogía con los vestidos que hacen ver linda a su hermana. Podemos interpretar que está enviando mensajes afectivos indirectos como: "encuentro que se ve lindo con su bufanda" (como su hermana con los vestidos que le hace su mamá); "yo lo quiero a usted y por eso no quiero que se resfríe" (el cuidado asociado al cariño). Estas formas indirectas de 
manifestar los afectos, desde los primeros años de vida, evidencian la amplitud del campo mental, mediante las relaciones analógicas y sus correspondientes desplazamientos metafóricos, como también la falta de herramientas de asertividad afectiva. Todo ello, necesario de incorporar en el proceso educativo desde la primera infancia.

\section{CATEGORÍA: INDETERMINACIÓN}

En la siguiente experiencia la educadora tiene planificado usar un cuento, pero decide cambiar la estrategia habitual que apuntaba a preguntar sobre los personajes y las secuencias de hechos una vez escuchada la historia. Ahora, antes de contar el cuento los lleva a imaginar y a verbalizar aquello que visualizan. Usa el cuento como una excusa para escuchar los propios mundos de los niños y niñas y sus formas de habitarlos.

- IPA19HK4 07/10/19

Educadora (Educ.)

Educ: ¿qué significará la palabra visualizar?

Niño 1: ¡ver!

Educ: ¡significa que uno va a imaginar y sentir que pasa en el cuento!

Niño 2: ¿puedo, en el libro, pasar como un cocodrilo?

Niño 3: ¡en mi casa imaginábamos que teníamos un helado!

Niña 1: yo me estaba imaginando en un barco

Niño 4: yo en el recreo tenía un pico de hierro y una espada de hierro

Niño 5: con un primo en la piscina imaginábamos que nos convertíamos en cosas, en animales

Niño 6: yo en mi casa, me convertía en una locomotora

Niña 2: yo en una nube volando

Educ: iy ustedes durante el cuento que hicieron?

Niña 3: escuchar, cerrar los ojos 
Educ: eso es lo que uno tiene que hacer cuando oye los cuentos, ir imaginando.

Niña 4: o cuando no sabes a qué jugar, ipuedes imaginar!

Educ: ¿qué más visualizaron?

Niño 7: ¡imaginamos que estábamos viendo un elefante!

Niña 5: ¡que estaban en la cueva de un oso!

Niño 8: ¡yo me subí a aun barco!

Niño 9: ¡cruzando un puente por debajo!

Niña 6: iyo imagine en el recreo que estaba en un rio!

Niño 10: ¡tia! porque ¿sabe?, que yo estaba imaginando que estoy en un auto y lo hago de verdad.

Niña 7: ¡yo manejaba un barco!

Niña 8: ¡yo afuera en el patio en un autobús!

Niño 11: ¡si! podíamos comer helado, y lo hicimos.

La educadora suelta el protagonismo sin saber que va a suceder. Esta didáctica, gatilló la expresión de diversas y creativas respuestas, porque los niños y niñas, tuvieron la oportunidad de pronunciar sus propias palabras; de participar, de escuchar y ser escuchado, sin un guion prestablecido; el poder, en estas relaciones, radica en la calidad de lo que comunican y no en las posiciones y roles jerárquicos de quienes pronuncian su palabra o constriñen el campo de respuesta de los otros(as). Lo que revela el registro, es precisamente que los niños y niñas van integrando los conocimientos cotidianos y escolares para poder expresar y explicitar sus propias ideas, situación que no ocurre cuando la experiencia educativa formal está planificada con tanta exhaustividad. La indeterminación abre posibilidades de exploración, descubrimiento, planteamiento de hipótesis, construcción de versiones distintas de lo que están conociendo; contrastaciones, defensas y contra argumentaciones sobre aquello. En la misma línea de análisis llegamos al aprendizaje dialógico, que considera que es precisamente en estas acciones donde se acorta la brecha social y cultural, poniendo en movimiento los principios de un aprendizaje desde las interacciones, es decir, movilizando la inteligencia cultural que crea sentido y aprendizajes más profundos y 
duraderos, posibles de ser transferidos a otros contextos, próximos o distales. La generación de instancias educativas requiere del diálogo igualitario, pues, al tener la oportunidad desde la edad más temprana de expresar el propio saber en un ambiente de respeto, contribuye al aprendizaje social-ciudadano, necesario para construir comunidad.

El siguiente registro (DIDULS, 2016), ayuda a ilustrar la idea, de lo indeterminado (emergente)

La educadora, les dice a los niños/as que armen la figura humana. Luego hace un recordatorio y dice: " $i d e l$ tronco salen las...?" y los niños y niñas completan diciendo: "ipiernas y brazos!; ¡las extremidades!".

- Stuart dice: “itía, tía (llama a la educadora) se me perdió una pierna!" (cada niño y niña tiene las partes del cuerpo humano que deben armar)

- Educadora dice: “ ¡Ah, nadie se mueva!, a Stuart se le perdió la pierna, ahora ¿cómo se va a ir a su casa?", niños y niñas ríen y la educadora pregunta: “¿A quién podemos llamar para que nos ayude?"

- Los niños responden: "¡a un doctor!"

- Luego Isidora dice: “ $¡ a$ Justin Bieber!”

- La educadora le dice: “¿Y en qué nos ayudaría él?"

- Isidora: “¿Eee, a curar la pierna, y a cantar para que divierta al enfermo!"

Podemos ver que la respuesta de Isidora es el hecho raro, es la respuesta no esperada, que surge de un contexto de alteridad donde no ve sólo un cuerpo sin una pierna, ella ve al ser humano que sufre y que requiere no sólo ayuda física, también ayuda emocional. Con su respuesta, que provocó más de alguna risa de quienes la escucharon, hace evidente la distancia entre la fragmentación/holismo, aspecto interesante de tener en cuenta al caracterizar la epistemología y la inteligencia cultural de las infancias en relación a la escolar. También es posible 92 | INTEREDU № 5 Vol. II (DiCIEMBRe 2021) PÁGS. 69-97. ISSN: 2735-6523 
considerar que, Isidora en situaciones previas en que se ha sentido triste, escuchar a Justin Bieber le haya generado alegría y en esta situación hipotética del compañero que perdió la pierna, proyecta esta experiencia como acto de humanidad, para ayudar al bienestar del enfermo, como ella lo denomina. Este hecho raro, pareciera no ser tal en el paradigma de la complejidad donde se aloja la epistemología infantil. En este caso, ella ha generado una relación entre la música y la cura de una dolencia, pero fundamentalmente ha visto a la persona en toda su dimensión humana.

Una respuesta afectiva, como la de Isidora, causa hilaridad y sorprende. Pero como la emocionalidad es parte constitutiva de nuestra naturaleza, no podemos negarla totalmente y emerge, por medio de microdiscursos como hace Pedro, usando expresiones metafóricas, utilizando imágenes sociales propias de sus contextos culturalescomunitarios extra-escuela.

Vemos que la alteridad, la empatía, la solidaridad y los afectos son esenciales para contribuir a una convivencia pacífica y saludable. El abordaje oportuno y el desarrollo temprano de estas habilidades van a depender de cuánto escuchamos y observamos a los niños y niñas, de qué valor le otorgamos a ello y del grado de desarrollo de las propias habilidades socio afectivas de los(as) educadores(as) y en general de las figuras significativas de su entorno.

En estos cortos diálogos que rompen la linealidad de lo planificado, niños y niñas ponen elementos reflexivos interesantes para responder a problemas lingüísticos, sociales, existenciales y educativos que, desde el punto de vista semiótico, podemos interpretar en relación al sentido de estos enunciados que emergen de un contexto o condición de producción determinada. Todos estos temas forman parte del conocimiento cotidiano de niños y niña y requieren mayor eco en el ambiente adulto, familiar y el escolar

\section{A MODO DE CONCLUSIÓN}

Diremos que el sistema educativo debe recuperar o reconocer otros lenguajes, otras competencias de tipo prácticas, culturales, emocionales y 
comunicativas para salir de la teoría del déficit tan fuertemente arraigada por la incidencia profunda del sistema academicista aun imperante en los sistemas educativos desde el nivel inicial. Centrarse en el déficit, lleva los(as) docentes a compensar dicha condición (Aubert et al., 2010), generando la disminución de sus expectativas en relación con las capacidades, competencias y habilidades de niños y niñas, afectando con ello su autoestima académica y personal. Un salto fundamental para la superación de esta tensión es transitar desde las pretensiones dialógicas a una práctica dialógica (Flecha, 2009).

Este estudio nos muestra la importancia de poner atención a las pedagogías invisibles y a los diálogos implícitos, porque los niños y niñas son traductores natos. Su capacidad para interpretar hasta el gesto más sutil, les lleva a ser sensibles al entorno y responder a la situación desplegando o replegando su deseo de aprender. En este sentido, nos hacen ver que la pedagogía debemos acercarla al mundo de la semiótica, para poner atención y reflexionar sobre los modos en que comprenden o expresan las cosas y ahondar en el plano filosófico desde donde nos hablan. Reconocer los mecanismos que utilizan para atribuir sentido a los contenidos escolares y sus diversas formas de representarlas, nos permite ver con mayor nitidez su epistemología. Este es el origen de los contextos educativos emergentes que están cargados de su cultura y de explicaciones, relatos y realidades que cambian, se modifican o complementan según sea el elemento de referencia con el que establecen algún tipo de relación. Para ello es indispensable transformar cualquier tipo de pedagogía que tienda a su "domesticación", por una, que permita la expansión del pensamiento, para propiciar el despliegue de su curiosidad y su capacidad de crear todos los mundos posibles.

Identificar esos mensajes presentes en estas pequeñas interacciones, nos ayuda en la tarea de ejercer el rol de agentes curriculares, para no perder de vista la valoración y el respeto por la epistemología propia de las personas en situación de aprendizaje (Rodrigo y Arnay, 1997). Estas situaciones que van develando la inteligencia cultural, por lo general, 94 | INTEREDU № 5 VOL. II (DiCIEMBRE 2021) PÁGS. 69-97. ISSN: 2735-6523 
quedan fuera del marco del currículo prescrito, por lo tanto, no se ven y como no se ven no existen, y si llegan a verse no hay un aprovechamiento claro o explícito de toda la riqueza semántica y axiológica que los niños y niñas van manifestando. Con ello podemos evidenciar que existe la necesidad de integrar a lo pedagógico y a lo organizativo, el sentimiento, la emoción, la intuición y la percepción connotativa, como una propuesta de acción que contribuya al cambio y a la mejora de la educación.

$\mathrm{Si}$ ponemos real atención a aquellos contextos emergentes, seguiremos encontrando luces que nos permitirán reconocer y acercar la inteligencia cultural de los niños y niñas para darle más sentido a la tarea educativa, porque es mediante este tipo de relaciones virtuales con una realidad paralela que emerge, que los niños y niñas van consolidando su aprendizaje y construyendo un conocimiento propio que se caracteriza por tener una naturaleza análoga con algún elemento concreto o virtual de un contexto. Esta cualidad flexible de los ambientes complejos e indeterminados es la que permite los flujos creativos, el protagonismo y la participación activa a los niños y niñas en los tiempo-espacios educativos.

REFERENCIAS BIBLIOGRÁFICAS

Acaso, M. (2018). Pedagogías Invisibles. El espacio del aula como discurso. Catarata

Aubert, A., Flecha,A., Flecha, R., García, C. y Racionero, S. (2010). Aprendizaje dialógico en la sociedad de la información. Hipatia editorial

Bateson, G. (1979). Espíritu y naturaleza. Ediciones WinuE

Bruner, J. (1999). La educación, puerta de la cultura. Visor.

Calvo, C. (2014). Asombros educativos infantiles y propensión a aprender. [Proyecto de investigación Fondecyt N¹110577]. Fondo Nacional de Investigación Científica y Tecnológica. 
Desirée López de Maturana, Miguel Valenzuela y Benjamín Álvarez

Catalán, J. (2010). Teorías Subjetivas. Aspectos teóricos y prácticos. Editorial Universidad La Serena.

Catalán, J. y Cuadra, D. (2016). Teorías subjetivas en profesores y su formación profesional. Revista brasileña de Educación. v. 21 n. 65. https://doi.org/10.1590/S1413-24782016216517

DIDULS (2016). Propensión a aprender y desarrollo de las funciones cognitivas. Departamento de Educación de la Universidad de La Serena.

Echeverría, R (2005). Ontología del lenguaje. Lom Ediciones S.A

Flecha, R (2009). Cambio, inclusión y calidad en las comunidades de aprendizaje. C \& E: Cultura y Educación, Num. 1, 157-170. ISSN 1135-6405

Freire, P. (1997). A la sombra de este árbol. Hipatia Editorial

Goldberg, E. (2006). La paradoja de la sabiduría. Drakontos

Gopnik, A (2010). El filósofo entre pañales. Ediciones Planeta

Krause, M. (1995). La investigación cualitativa - Un campo de posibilidades y desafíos. Revista: Temas de educación N7, 19-39. ISSN 0716-7423-19

L'Ecuyer, C (2012) Educar en el asombro. Plataforma

López de Maturana, S. (2014). Los Buenos Profesores: Educadores comprometidos con un proyecto educativo. Editorial Universidad de La Serena.

López de Maturana, S. (2010). Maestros en el Territorio. Editorial Universidad de La Serena.

Madrona, S. (2014, 11 de abril). El yo es un concepto con fecha de caducidad. Tendencias21. https://tendencias21.levante-emv.com/elyo-es-un-concepto-con-fecha-de-caducidad_a32818.html 
Maturana, H. (2001). Emociones y lenguaje, en educación y política. Dolmen.

Rodrigo, M. y Arnay, J. (1997). La construcción del conocimiento escolar. Temas de Psicología, Paidós, (1). ISBN 84-493-0358-3.

Scarpa, R (1978). Gabriela piensa en la madre ausente. Andrés Bello

Taleb, N. (2011). El cisne negro. El impacto de lo altamente improbable. Paidos

Varela, F. (2000). El fenómeno de la vida. Cuatro pautas para el futuro de las ciencias cognitivas. Dolmen.

Wolff, M. (1994). La cotidianeidad. Ediciones Cátedra. 